\title{
Two cases of paediatric autoimmune neuropsychiatric disorder associated with group A beta haemolytic streptococcal infection
}

\author{
Hemamali Perera ${ }^{1}$, Malika Weerasinghe ${ }^{2}$, Geetha M Sathiadas ${ }^{3}$
}

Sri Lanka Journal of Child Health, 2010; 39:29-30

(Key Words: PANDAS, Sydenham chorea, tic disorder, Group A haemolytic streptococcal infection)

Prominent neurological and/or psychiatric sequelae occur in a small percentage of children with streptococcal infection. Of these, Sydenham chorea (SC) is the best recognized syndrome. Paediatric autoimmune neuropsychiatric disorders associated with group A beta haemolytic streptococcal infection (PANDAS) is also a well-defined syndrome in which motor and/or vocal tics and/or obsessive-compulsive disorder (OCD) are the main clinical features ${ }^{1}$.

\section{Case 1}

S.A., a 9 year old boy, presented with involuntary jerky movements of head, neck and shoulder regions of 4 months duration. Onset of symptoms was sudden and persisted at the same intensity throughout. There were no involuntary vocal utterances, excessive eye blinking or jerky movements elsewhere in the body. His development was age appropriate. S.A. had a throat infection 4 weeks before onset of symptoms, and had treatment with antibiotics. Neck movements similar to that in the current episode occurred 18 months previously, lasted 2 weeks, and disappeared without treatment. Parents were uncertain whether that episode too was preceded by a throat infection. There was no family history of movement disorders or psychiatric illness. Results of laboratory investigations were as follows: ESR $9 \mathrm{~mm} / 1 \mathrm{st}$ hour; blood picture - red blood cells were normocytic normochromic, white blood cell count $7.3 \times 10^{9} / \mathrm{L}$ with neutrophils $49 \%$ lymphocytes $41 \%$ eosinophils $2.8 \%$; ECG - normal rate and rhythm; echocardiogram - small ASD $5 \mathrm{~mm}$ in size with a left to right shunt and awaiting review in 1 year; throat swab - beta haemolytic streptococci not isolated; ASOT - initially $800 \mathrm{IU} / \mathrm{ml}$, and $400 \mathrm{IU} / \mathrm{ml}$ two weeks later. Treatment was oral penicillin $250 \mathrm{mg} 6$ hourly for 2 weeks. Tics were treated with

\footnotetext{
${ }^{1}$ Professor in Psychological Medicine, University of Colombo, ${ }^{2}$ Registrar in Psychiatry, ${ }^{3}$ Senior Registrar in Paediatrics, Lady Ridgeway Hospital for Children, Colombo.
}

(Received on 20 October 2008. Accepted on 23 January 2009) risperidone $1 \mathrm{mg} /$ day with marked reduction of tic frequency at one month follow up.

\section{Case 2}

M.K., a 9 year old boy presented with a history of sudden onset jerky involuntary movements of the head and neck of 2 weeks duration. Movements were frequent, present throughout the day with associated pain. There was no facial grimacing, excessive blinking or involuntary vocal utterances. There was no past history of similar abnormal movements. There was a history of recent throat infection but no tonsillitis. No family history of tics or psychiatric disorder. The development was age appropriate. Laboratory investigation results were as follows: ESR $4 \mathrm{~mm} / 1 \mathrm{st}$ hour; blood picture - red blood cells were normocytic normochromic, white blood cell count $10.1 \times 10^{9} / \mathrm{L}$ with neutrophils $20 \%$ lymphocytes $72 \%$ eosinophils $8 \%$; ECG - normal rate and rhythm; echocardiogram - no abnormalities; throat swab - beta hemolytic streptococci were not isolated; ASOT - initially $400 \mathrm{IU} / \mathrm{ml}$ and remained at $400 \mathrm{IU} / \mathrm{ml}$ two weeks later. Treatment was oral penicillin $250 \mathrm{mg} 6$ hourly for 2 weeks. Tics were treated with risperidone $1 \mathrm{mg} /$ day with marked reduction of tic frequency at one month follow up.

\section{Discussion}

Published research guidelines for diagnosis of PANDAS specify the presence of a tic disorder and/or obsessive compulsive disorder; prepubertal age at onset between 3 and 12 years; abrupt onset and /or episodic course of symptom severity; temporal association between symptom exacerbations and streptococcal infection, confirmed by positive culture and / or elevated anti streptococcal titres; and the presence of neurological abnormalities during periods of symptom exacerbation ${ }^{2}$. Both our patients fulfilled the diagnostic criteria except for a positive culture for streptococcal infection, probably as a result of treatment with antibiotics prior to diagnosis. Tics, but not OCD was diagnosed in our patients, though the two may occur together. 
Autoimmune antibodies to basal ganglia are implicated in the aetiology of PANDAS and the onset of symptoms of tics and $\mathrm{OCD}^{2}$. It is hypothesized that the pathogenic process is triggered by an abnormal immune response to streptococcal infection in a susceptible host. Imaging studies identify a distinct subgroup of subjects with OCD and/or tics who have enlarged basal ganglia, thereby supporting this hypothesis ${ }^{3}$.

Close similarities with SC may imply that there is a potential for carditis in PANDAS too. However, carditis or valvular disease has not been demonstrated in PANDAS ${ }^{4}$. Accordingly, the available treatment guidelines specify that prophylactic antibiotics are not indicated ${ }^{4,5}$. Also, penicillin prophylaxis was found to be ineffective in preventing exacerbation of tics and OCD symptoms in PANDAS ${ }^{6}$. Thus, PANDAS is identified as a clinical entity distinct from $\mathrm{SC}^{1}$.

\section{References}

1. Swedo SE, Leonard HL, Garvey MA, et al. Pediatric autoimmune neuropsychiatric disorders associated with streptococcal infections: clinical description of the first 50 cases. American Journal of Psychiatry 1998; 155: 264 -71.
2. Swedo E, Leonard HL. The paediatric autoimmune neuropsychiatric disorders associated with streptococcal infection (PANDAS) subgroup: separating facts from fiction. Paediatrics 2004; 113: 907-11.

3. Giedd JN, Giedd JN, Rapoport JL, Garvey MA, Perlmutter S, Swedo SE. MRI assessment of children with obsessive-compulsive disorder and tics associated with streptococcal infection. American Journal of Psychiatry 2000; 157: 281-3.

4. Snider LA, Snider LA, Sachdev V, MacKaronis JE, St. Peter M, Swedo SE. Echocardiographic findings in the PANDAS subgroup. Pediatrics 2004; 114: 748-51.

5. Practice parameters for the assessment and treatment of children and adolescents with obsessive-compulsive disorder. Journal of the American Academy of Child and Adolescent Psychiatry 1999; 38: 499 -500.

6. Garvey MA, Perlmutter SJ, Allen AJ, Hamburger S, Lougee L, Leonard HL, et al. A pilot study of penicillin prophylaxis for neuropsychiatric exacerbations triggered by streptococcal infections. Biological Psychiatry 1999; 45:1564-71. 